Article

\title{
Statistical Model-Based Classification to Detect Patient-Specific Spike-and-Wave in EEG Signals
}

\author{
Antonio Quintero-Rincón ${ }^{1,2}, *\left(\mathbb{0}\right.$, Valeria Muro ${ }^{2}$, Carlos D'Giano ${ }^{2}$, Jorge Prendes ${ }^{3}(\mathbb{C}$ \\ and Hadj Batatia 4 (D) \\ 1 Departament of Electronic, Catholic University of Argentina (UCA), Av. Alicia Moreau de Justo 1300, \\ Buenos Aires C1107AAZ, Argentina \\ 2 Foundation for the Fight against Pediatric Neurological Disease (FLENI), Montañeses 2325, \\ Buenos Aires C1428AQK, Argentina; vmuro@fleni.org.ar (V.M.); cdigiano@fleni.org.ar (C.D.) \\ 3 IRIT-INPT-ENSEEIHT, University of Toulouse, 31000 Toulouse, France; jorge.prendes@tesa.prd.fr \\ 4 MACS School, Heriot-Watt University, Dubai Campus, Dubai Knowledge Park, Blocks 5 \& 14, Dubai 38103, \\ UAE; h.batatia@hw.ac.uk \\ * Correspondence: antonioquintero@uca.edu.ar
}

Received: 11 September 2020; Accepted: 27 October 2020; Published: 29 October 2020

\begin{abstract}
Spike-and-wave discharge (SWD) pattern detection in electroencephalography (EEG) is a crucial signal processing problem in epilepsy applications. It is particularly important for overcoming time-consuming, difficult, and error-prone manual analysis of long-term EEG recordings. This paper presents a new method to detect SWD, with a low computational complexity making it easily trained with data from standard medical protocols. Precisely, EEG signals are divided into time segments for which the continuous Morlet 1-D wavelet decomposition is computed. The generalized Gaussian distribution (GGD) is fitted to the resulting coefficients and their variance and median are calculated. Next, a $k$-nearest neighbors $(k-\mathrm{NN})$ classifier is trained to detect the spike-and-wave patterns, using the scale parameter of the GGD in addition to the variance and the median. Experiments were conducted using EEG signals from six human patients. Precisely, 106 spike-and-wave and 106 non-spike-and-wave signals were used for training, and 96 other segments for testing. The proposed SWD classification method achieved 95\% sensitivity (True positive rate), 87\% specificity (True Negative Rate), and 92\% accuracy. These promising results set the path for new research to study the causes underlying the so-called absence epilepsy in long-term EEG recordings.
\end{abstract}

Keywords: spike-and-wave; generalized Gaussian distribution; EEG; Morlet wavelet; $k$-nearest neighbors classifier; epilepsy

\section{Introduction}

Epilepsy is a chronic neurological disorder that affects patients, causing recurrent seizures. Seizures are characterized by excessive electrical discharges in neurons. Their waveform, known as the spike, is characterized by brief bursts of high amplitude, synchronized and multiphasic activity with several polarity changes [1]. These are exhibited close to the epileptic focus and stand out from the background EEG activity. Electroencephalography (EEG) is currently the main technique to record electrical activity in the brain. Neurologists, trained in EEG, are able to properly determine an epilepsy diagnosis by analyzing the different types of spikes in the so-called rhythmic activity of the brain.

Existing, automatic methods for detecting epileptic events in EEG signals have performance that greatly exceed visual inspection. These methods focus mostly on interictal spikes [2,3], seizure onset detection [4], or waveform epileptic patterns [5,6]. There exists a wide variety of methods to accurately detect seizures and their patterns in EEG. Most of these methods are based on supervised 
machine learning techniques, such as Support Vector Machine [7], logistic regression [8], decision trees [9], $k$-Nearest Neighbor, Random Forest [10], or discriminant analysis [11]. They mainly differ according to their feature extraction and classification approaches. A large variety of features are used, including spatio-temporal analysis [12], spectral-temporal analysis [13], wavelet decomposition [2], spectrogram [14,15], Hilbert transform [16], neural networks [17], Hurst exponent [18], quadratic linear-parabolic model [19], and statistical descriptors such as statistical modeling [20], signal fuzzy entropy [21], and fractal dimension [22]. The reader is referred to [23] for a recent state-of-the-art on methods for seizure detection in EEG.

Spike-and-wave discharge (SWD) is a generalized EEG discharge pattern, where the waveform has a regular and symmetric morphology. This morphology can be mathematically described by a Morlet wavelet transform, generating a time-frequency representation of the EEG signal [24-27]. The spike component of an SWD is associated with neuronal firing and the wave component is associated with neuronal inhibition or hyperpolarization of neurons [28]. SWD is widely used in mice studies $[2,14,29,30]$; inversely, the literature reports very limited human applications. Mice have a predisposition for generalized SWD at 7-12 Hz [15]. Typically, they have spontaneous absence-seizure-like events, but, the presence of an intact cortex, thalamus, and their interconnections are necessary to record their signals [31,32]. Rodent models are usually used to study the neurobiological mechanisms underlying SWD in humans. However, studies in humans and rodents differ in the way SWD is assessed and, more importantly, rodents and humans show substantial biological differences. Thus, more human studies are necessary to fully understand this phenomena. This paper fits in this general aim by studying SWD in human.

Some recent works have been proposed to estimate SWD patterns in humans using machine learning techniques. They rely on different models and features, including 1-NN with t-location-scale distribution [20], decision-trees with cross-correlation coupled with decision trees [9], and Bayesian classifiers with the Walsh transformation [33]. Table 1 lists some more methods. Other existing methods implement signal analysis techniques, such as Hilbert-Huang transform to analyze time-frequency energy distribution [34], complex network of neuronal oscillators to model SWD [35], analyzing statistical features such as variance, the sum of wave amplitudes, slope of the wave [36], or topographic cluster analysis based on connectivity, entropy, frequency, power, and spike amplitude [37]. For a biological dynamic explanation of features and mechanisms generating SWD in the brain see [38].

Table 1. Some state-of-the-art methods for the spike-and-wave discharge (SWD) estimation in electroencephalography (EEG) signals in humans, compared in terms of classification techniques, features, and reported performance.Abbreviations are as follows: high Specificity, rule in (SpPIn), According to the frequency and magnitude weighted average (WA), According to an estimated threshold (AET).

\begin{tabular}{|c|c|c|c|c|}
\hline Method & Features & Classifier & Accuracy in \% & Ref. \\
\hline $\begin{array}{l}\text { Generalized Gaussian distribution } \\
\text { (GGD) }\end{array}$ & $\begin{array}{l}\text { GGD parameters, variance and median } \\
\text { from time-frequency Morlet decomposition }\end{array}$ & $10-\mathrm{NN}$ & 92 & our \\
\hline Kendall's Tau-b Coefficient & Kendall's Tau-b coefficient significance & SpPIn & 94 & [39] \\
\hline Ramanujan Filter Bank (RFB) & Spectrum from RFB & Empirical & $>80$ & [40] \\
\hline t-location-scale distribution (TLS) & TLS parameters & $1-\mathrm{NN}$ & 100 & [20] \\
\hline Cross-correlation & Correlation coefficient & Decision trees & 97 & [9] \\
\hline Walsh transformation (WT) & First and second orden from WT & Bayesian & $>70$ & [33] \\
\hline Hilbert-Huang transform & Intrinsic mode functions energy & WA & - & [34] \\
\hline Cross-correlation & Wavelet spectrum correlation & AET & 100 & [41] \\
\hline
\end{tabular}

This paper presents a new SWD patient-specific detection method based on the statistical modeling of the continuous Morlet wavelet coefficients. Precisely, we fit the generalized Gaussian distribution to these coefficients and estimate the corresponding parameters. These parameters are used as features in a 10-NN classifier. Training and testing of the learning model use different EEG datasets.

The remainder of the paper is structured as follows. Section 2 presents the EEG database, and the proposed methodology, where we explain the continuous Morlet wavelet transformation, 
the generalized Gaussian distribution (GGD) statistical model, and the $k$-NN classifier. Experimental results using the scale parameter from the GGD and the variance and median from the continuous wavelet coefficients are reported in Section 3, flowed by discussion in Section 4. Conclusions, remarks, and perspectives are presented in Section 5.

\section{Materials and Methods}

This section presents our statistical model-based method to detect spike-and-wave discharges (SWD) in EEG signals. It is computationally very efficient, suitable for real-time implementation, allowing on-line spike-and-wave detection. First, we describe the dataset used for experimentation, then we present the different processing steps.

\subsection{Database}

A standard 10/20 EEG system was used at the Foundation for the Fight against Pediatric Neurological Disease (FLENI) to acquire long-term $256 \mathrm{~Hz}$ EEG signal, from 12 human patients. The following 22-channels were used: Fp1, Fp2, F7, F3, Fz, F4, F8, T3, C3, Cz, C4, T4, T5, P3, Pz, P4, T6, $\mathrm{O} 1, \mathrm{O} 2, \mathrm{Oz}, \mathrm{FT10}$ and FT9. See [39] for more details. The acquired signals had a different waveform and duration.

All EEG signals were labeled by a neurologist from FLENI to indicate the onset and duration of the epilepticform. Based on these annotations, we extracted 212 short epochs (1-min average duration) focusing on the spike-and-wave waveform. As such, a database with the 212 monopolar signal epochs was created, with 106 SWD signals and 106 non-SWD signals. Each SWD signal has been restricted to a narrow frequency band between 1-3 Hz. Figure 1 shows an example of a typical SWD signal. Visually, one can observe that SWD patterns exhibit characteristic morphology; whereas non-SWD signals have normal waveform.

Six patients with twenty (20) signals each were used for training our predictive model (Section 2.2). This multiplicity of signals from the same patient has been decided to enforce learning-patient specific patterns. A set of 96 signals from the other six patients were used for testing. 

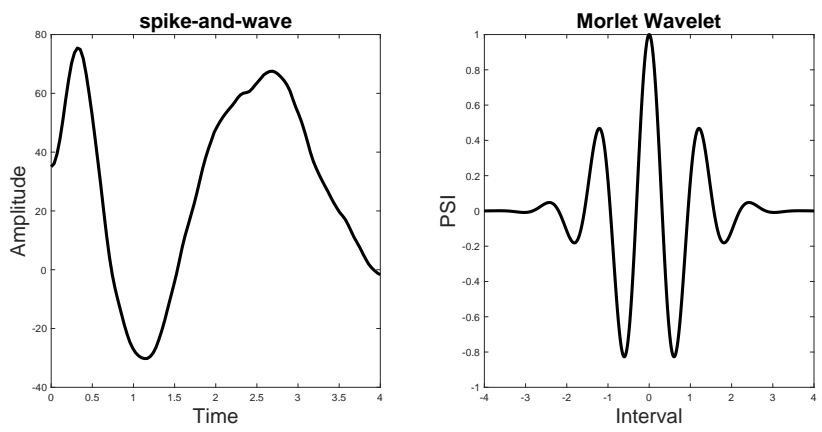

(a) SWD and Morlet Wavelet

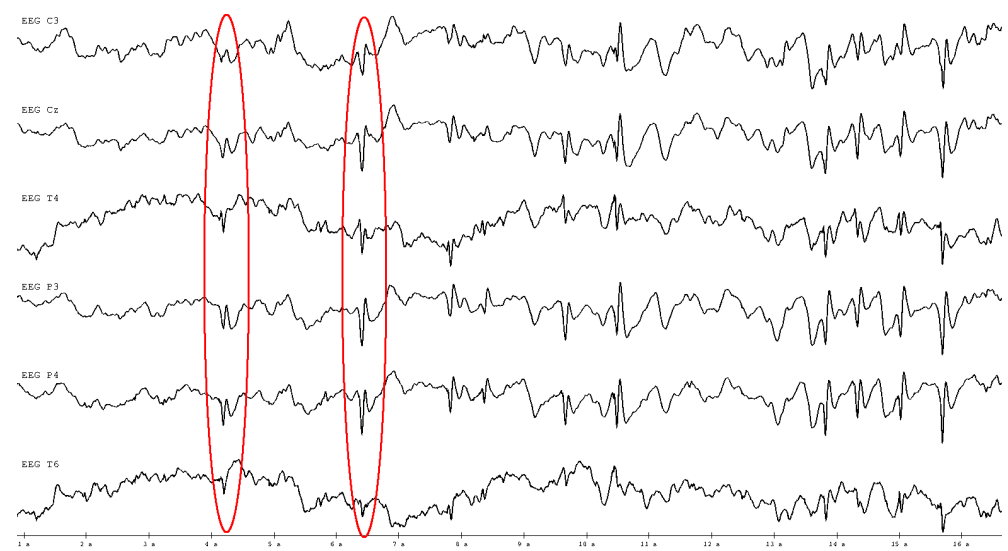

(b) EEG example

Figure 1. (a) A sample SWD signal and the Morlet wavelet. Note that the scales are different. (b) Example of 6 channels of one monopolar raw EEG, with SWD patterns in all channels.

\subsection{Methodology}

The proposed method is composed of four stages. The first stage divides every EEG signal epoch, $X \in \mathbb{R}^{N \times M}$ with $M$ channels and $N$ time instants, into two-second segments per channel, with one-second overlap across a rectangular sliding window. Please note that $M$ is fixed to 22, whereas $N$ varies for different epochs (with an average duration of $60 \mathrm{~s}$ giving about $N=256 * 60=15,360$ samples). This will give [N/60] segments per channel. The second stage consists of applying Morlet decomposition to create separate time-frequency representations for each segment $\boldsymbol{X}_{t} \in \mathbb{R}^{N \times 1}$. The purpose of this decomposition is to evaluate the energy distribution throughout the SWD frequency band $(1-3 \mathrm{~Hz})$. In the third stage, the statistical distribution of the wavelet coefficients from each segment is represented using a zero-mean generalized Gaussian distribution (GGD). A maximum likelihood method is used to estimate the GGD parameters, scale ( $\varsigma$ ) and shape $(\tau)$ [4,42-44]. This statistical modeling stage gives $M \times[N / 60]$ pairs of scale $(\varsigma)$ and shape $(\tau)$ parameters, achieving a very strong dimension reduction. As we demonstrate it in the experimentation section, the scale parameter $\zeta$ was found statistically characteristic of the SWD waveform, and it is proposed as a feature to detect such patterns $[4,44]$. Using a single parameter to classify patterns is too strict and misses the natural variability in the data. For this reason, we compute two other statistical parameters, namely the variance $\left(\sigma^{2}\right)$ and the median $(\widetilde{x})$ from the wavelet coefficients of each segment. The data from one EEG epoch reduces therefore to three parameters, $\left\{\varsigma, \sigma^{2}, \widetilde{x}\right\}$, giving a high dimension reduction while offering a flexible representation space to discriminate patterns while accounting for natural variability. Please note, in total, we will have $M \times[N / 60] * 3$ parameters for any EEG signal epoch. Finally, a classification model has been trained to detect SWD patterns, using the three features parameters. The proposed methodology is summarized in Figure 2. The following sections describe each stage. 
We now introduce the Morlet wavelet, the Generalized Gaussian distribution, and the k-nearest neighbor classifier used in this paper.

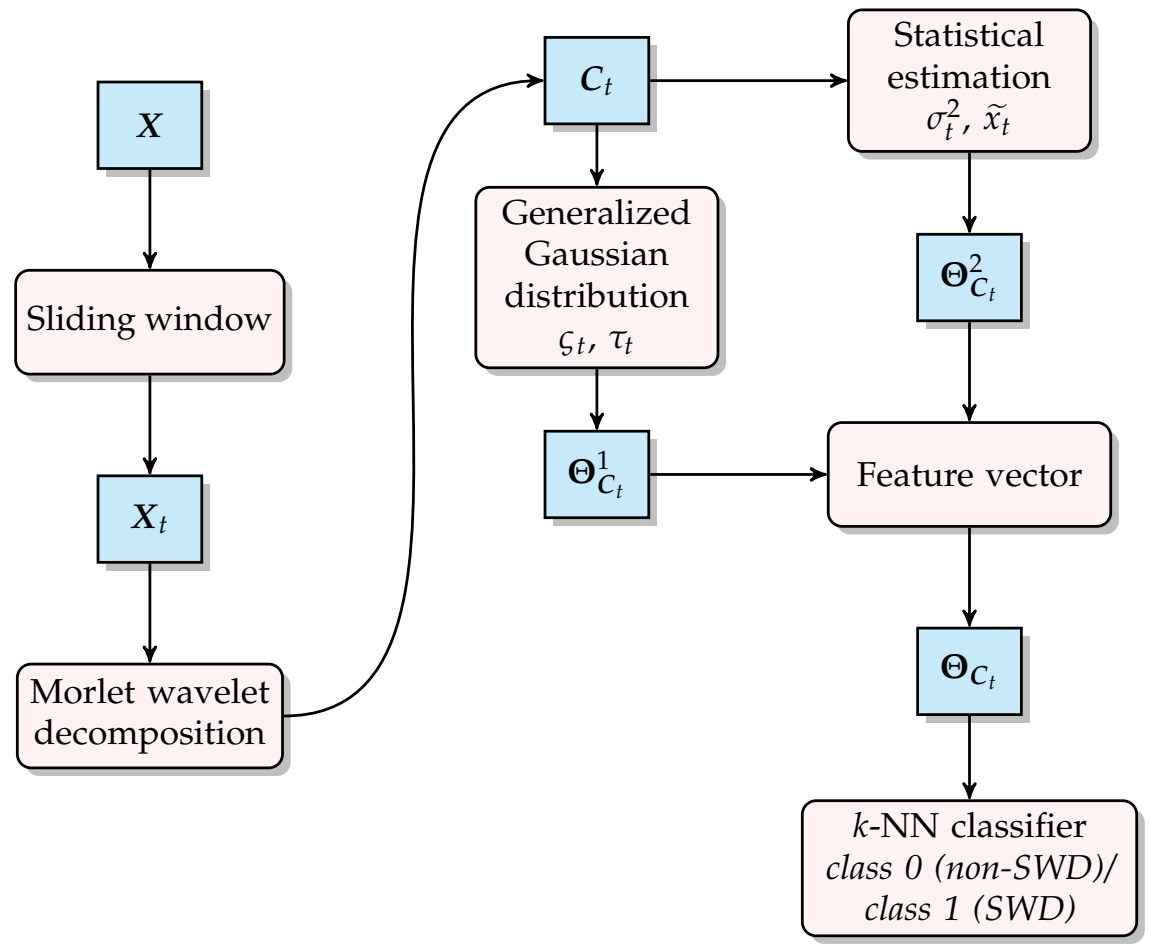

Figure 2. Diagram of the proposed method.

\subsubsection{Morlet Wavelet}

The continuous wavelet transform is given by

$$
\begin{aligned}
W_{f}(t, a, b) & =\int_{-\infty}^{\infty} \boldsymbol{X}_{t} \psi_{a, b}^{*}(t) \mathrm{dt} \\
\psi_{a, b}^{*}(t) & =\frac{1}{\sqrt{a}} \psi\left(\frac{t-b}{a}\right) \\
\psi(t) & =\exp ^{-\frac{t^{2}}{2}} \cos (5 t)
\end{aligned}
$$

where $a$ is the scaling parameter and $b$ the shift parameter. Equation (2) is the mother wavelet function, where $(*)$ denotes the complex conjugate operation. Equation (3) is the analytic expression of the Morlet wavelet [45]. In order to use the Morlet wavelet with frequency $f_{c}$, we use the relationship

$$
f_{a}=\frac{f_{c}}{\alpha \Delta}
$$

where $\alpha$ is the scale, $\Delta$ is the sampling period, $f_{c}$ is the center frequency of Morlet wavelet (in $\mathrm{Hz}$ ) and $f_{a}$ is the pseudo-frequency corresponding to the scale $a$ (in $\mathrm{Hz}$ ). As such, the wavelet dominant frequency can be characterized using the center frequency, as it detects the main wavelet oscillations (the reader is referred to [46] for details). Note that the wavelet scale is estimated according to the narrow frequency $1-3 \mathrm{~Hz}$ from (Section 2.1). In our case, $\Delta=256$, and $1<$ frequency $<3$, we have a scale $\alpha \in f_{c} * \Delta, f_{c} * \Delta / 3$. Figure 3 show an example of time-scale variation for a value of $f_{\mathcal{c}}=1.2308$ for SWD and $f_{c}=0.8125$ for non-SWD. These data are from signal 1 from patient 1 . 


\subsubsection{Generalized Gaussian Distribution}

The generalized Gaussian distribution (GGD) is a flexible statistical model often used in science and engineering to represent data. We propose to represent the distribution of the Morlet wavelet coefficients $\left(\boldsymbol{C}_{t}\right)$ using the GGD [47]. The probability density function (PDF) of the GGD is given by the expression

$$
f_{\mathrm{GGD}}(x ; \zeta, \tau)=\frac{\tau}{2 \varsigma \Gamma\left(\tau^{-1}\right)} \exp \left(-\left|\frac{x}{\zeta}\right|^{\tau}\right)
$$

where $\varsigma \in \mathbb{R}^{+}$is a scale parameter, $\tau \in \mathbb{R}^{+}$is a shape parameter, and $\Gamma(\cdot)$ is the Gamma function. Fitting equation (5) to the data $C_{t}$ is performed using the maximum likelihood estimators $\Theta_{C_{t}}^{1}$ :

$$
\boldsymbol{\Theta}_{\mathcal{C}_{t}}^{1}=\left[\varsigma_{t}, \tau_{t}\right]^{T}=\underset{[\zeta, \tau]^{T}}{\arg \max } f_{\mathrm{GGD}}\left(\boldsymbol{C}_{t} ; \zeta, \tau\right)
$$

For more details about the estimation of the GGD parameters, we refer the reader to our previous work $[4,42-44,48]$.

\subsubsection{Feature Parameters}

Through the previously-described stages, data from every signal epoch, $M \times N$ samples, is reduced to the matrix of parameters (or features) $\boldsymbol{\Theta}_{C_{t}}$, composed of $M \times[N / 60]$ rows, with three columns consisting of our data, reduced to the matrix of parameters (or features) $\boldsymbol{\Theta}_{C_{t}}$, composed of the scale parameter from the GGD, the variance and the median of the Morlet wavelet coefficients.

$$
\boldsymbol{\Theta}_{C_{t}}=\left[\zeta_{t}, \sigma_{t}^{2}, \widetilde{x_{t}}\right]
$$

Using this representation, the next stage consists of training a $k$-nearest neighbors classifier to detect SWD patterns. Please, recall that we have 212 signal epochs in our database. All went through the preceding dimension reduction process. A set of 120 of those (from six patients) served for training and the remaining 96 (from the six other patients) were used for testing. 


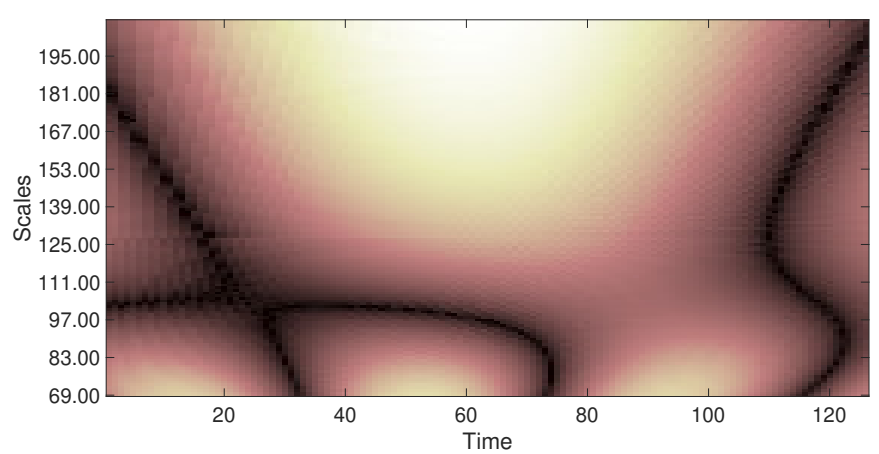

(a) SWD

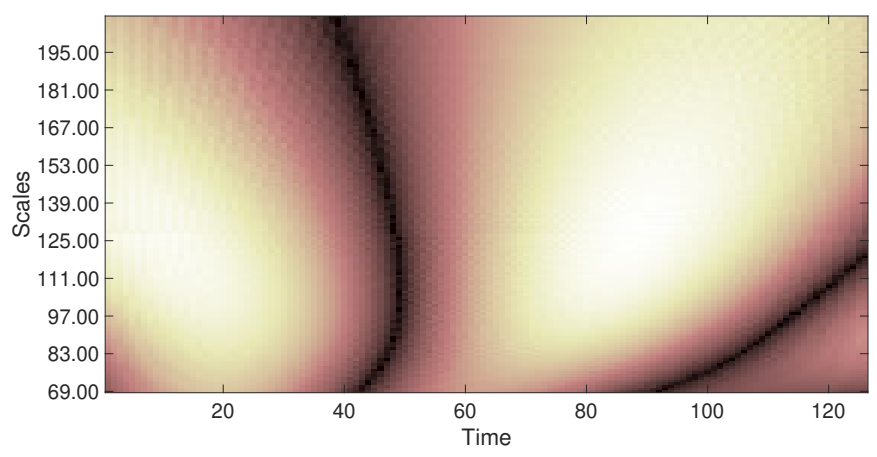

(b) non-SWD

Figure 3. Illustration of the variation of time-scale for Morlet continuous wavelet (a) for SWD with $f_{c}=1.2308 \mathrm{~Hz}$, and (b) for non-SWD with $f_{c}=0.8125$. Note that, the energy distribution pattern is different when comparing SWD and non-SWD.

\subsection{4. $k$-Nearest Neighbors Classification}

Using the feature vector $\boldsymbol{\Theta}_{C_{t}}$, consider a classification into two possible classes $c=0$ (non-SWD) and $c=1$ (SWD). The probability of classifying a sample in one of the two classes is given by

$$
\begin{aligned}
\rho\left(\boldsymbol{\Theta}_{C_{t}} \mid c=0\right) & =\frac{1}{N_{0}} \sum_{n \in \text { class } 0} \mathcal{N}\left(\Theta_{C_{t}} \mid \Theta_{C_{t}}^{n} \sigma^{2} \boldsymbol{I}\right) \\
& =\frac{1}{N_{0}\left(2 \pi \sigma^{2}\right)^{D / 2}} \sum_{n \in \text { class } 0} \exp ^{-\frac{\left(\boldsymbol{\Theta}_{C_{t}}-\boldsymbol{\Theta}_{C_{t}}^{n}\right)^{2}}{2 \sigma^{2}}} \\
\rho\left(\boldsymbol{\Theta}_{C_{t}} \mid c=1\right) & =\frac{1}{N_{1}} \sum_{n \in \text { class } 1} \mathcal{N}\left(\boldsymbol{\Theta}_{C_{t}} \mid \boldsymbol{\Theta}_{C_{t^{\prime}}}^{n}, \sigma^{2} \boldsymbol{I}\right) \\
& =\frac{1}{N_{1}\left(2 \pi \sigma^{2}\right)^{D / 2}} \sum_{n \in \text { class } 1} \exp ^{-\frac{\left(\boldsymbol{\Theta}_{C_{t}}-\Theta_{C_{t}}^{n}\right)^{2}}{2 \sigma^{2}}},
\end{aligned}
$$

where $D$ is the dimension of the sammple $\Theta_{C_{t}}, N_{0}$ and $N_{1}$ are the numbers of training samples from class 0 and class 1 , respectively; and $\sigma^{2}$ is the variance. Using the Bayes rule to classify a new observation $\boldsymbol{\Theta}_{C_{t}}^{*}$, we obtain the following equation

$$
\rho\left(c=0 \mid \boldsymbol{\Theta}_{C_{t}}^{*}\right)=\frac{\rho\left(\boldsymbol{\Theta}_{C_{t}}^{*} \mid c=0\right) \rho(c=0)}{\rho\left(\boldsymbol{\Theta}_{C_{t}}^{*} \mid c=0\right) \rho(c=0)+\rho\left(\boldsymbol{\Theta}_{C_{t}}^{*} \mid c=1\right) \rho(c=1)}
$$

The maximum likelihood gives $\rho(c=0)=N_{0} /\left(N_{0}+N_{1}\right)$, and $\rho(c=1)=N_{1} /\left(N_{0}+N_{1}\right)$. Substituting in Equation (10), we obtain the probability $\rho\left(c=0 \mid \Theta_{\mathcal{C}_{t}}^{*}\right)$. The expression for $\rho\left(c=1 \mid \Theta_{\mathcal{C}_{t}}^{*}\right)$ can be 
derived in a similar manner. To determine which class is most likely, the ratio between the two expressions is evaluated

$$
\frac{\rho\left(c=0 \mid \Theta_{C_{t}}^{*}\right)}{\rho\left(c=1 \mid \Theta_{C_{t}}^{*}\right)}=\frac{\rho\left(\boldsymbol{\Theta}_{C_{t}}^{*} \mid c=0\right) \rho(c=0)}{\rho\left(\boldsymbol{\Theta}_{C_{t}}^{*} \mid c=1\right) \rho(c=1)}
$$

If the ratio is greater than one, $\boldsymbol{\Theta}_{C_{t}}^{*}$ is classified as $c=0$, otherwise it is classified as $c=1$. It is important to note that in the case where $\sigma^{2}$ is very small in (11), then both the numerator and denominator will be dominated by the term for which the sample $\Theta_{C_{t}}^{n_{0}}$ in class- 0 or $\Theta_{C_{t}}^{n_{1}}$ in class- 1 are closest to the point $\Theta_{C_{t}}^{*}$, such that

$$
\begin{aligned}
& \frac{\rho\left(c=0 \mid \boldsymbol{\Theta}_{C_{t}}^{*}\right)}{\rho\left(c=1 \mid \boldsymbol{\Theta}_{C_{t}}^{*}\right)}=\frac{\exp ^{-\frac{\left(\boldsymbol{\Theta}_{C_{t}}^{*}-\boldsymbol{\Theta}_{C_{t}}^{n_{0}}\right)^{2}}{2 \sigma^{2}}} \rho(c=0) / N_{0}}{\exp -\frac{\left(\boldsymbol{\Theta}_{C_{t}}^{*}-\boldsymbol{\Theta}_{C_{t}}^{n_{1}}\right)^{2}}{2 \sigma^{2}} \rho(c=1) / N_{1}} \\
& =\frac{\exp ^{-\frac{\left(\Theta_{C_{t}}^{*}-\Theta_{C_{t}}^{n_{0}}\right)^{2}}{2 \sigma^{2}}}}{\exp ^{-\frac{\left(\Theta_{C_{t}}^{*}-\Theta_{C_{t}}^{n_{1}}\right)^{2}}{2 \sigma^{2}}}}
\end{aligned}
$$

On the limit $\sigma^{2} \rightarrow 0, \boldsymbol{\Theta}_{\mathcal{C}_{t}}^{*}$ is classified as class 0 if $\boldsymbol{\Theta}_{\mathcal{C}_{t}}^{*}$ has a point in the class 0 data which is closer than the closest point in the class 1 data. The nearest neighbor method is therefore recovered as the limiting case of a probabilistic generative model. The parameter $k$ is chosen based on $\sqrt{N}$, where $N$ is the number of samples in the training dataset. We refer the reader to $[49,50]$ for a comprehensive treatment of the mathematical properties of $k$-nearest neighbors classifier.

\section{Results}

The annotated database introduced in Section 2.1 was used to compute the feature vector $\left[\varsigma_{t}, \sigma_{t}^{2}, \widetilde{x}_{t}\right] \in \mathbb{R}^{3}$, based on the statistical model of the coefficients of the continuous Morlet wavelet. The resulting features were used for off-line training the $k$-nearest neighbor classifier. With the 212 samples, $k$ was set to 10 giving a 10 -nearest neighbor.

Tables $2-4$ show the statistical mean, standard deviation, variance and bounds values from the feature vector. One can note that, sigma $\left(\varsigma_{t}\right)$, variance $\left(\sigma_{t}^{2}\right)$, and median $\left(\widetilde{x}_{t}\right)$ are larger for SWD that for non-SWD. Therefore, despite the overlapping statistical bounds, a threshold can be determined to detect SWD patterns.

Table 2. Mean, standard deviation, variance and bound values for sigma parameter $(\varsigma)$ for class 0 (non-spike-and-wave) and class 1 (spike-and-wave).

\begin{tabular}{ccccc}
\hline & Mean & std & Variance & Bounds \\
\hline Class 0 & 293 & 267.8017 & 71,718 & {$[12,1275]$} \\
Class 1 & 542 & 406.2597 & 165,047 & {$[31,1811]$} \\
\hline
\end{tabular}

Table 3. Mean, standard deviation, variance and bound values for the variance $\left(\sigma_{t}^{2}\right)$ for class 0 (non-spike-and-wave) and class 1 (spike-and-wave).

\begin{tabular}{ccccc}
\hline & Mean & std & Variance & Bounds \\
\hline Class 0 & $1.446 \times 10^{6}$ & $4.235 \times 10^{6}$ & $1.794 \times 10^{13}$ & {$\left[9.46 \times 10^{2}, 3.162 \times 10^{7}\right]$} \\
Class 1 & $4.32 \times 10^{6}$ & $7.892 \times 10^{6}$ & $6.228 \times 10^{13}$ & {$\left[2.715 \times 10^{3}, 4.321 \times 10^{7}\right]$} \\
\hline
\end{tabular}


Table 4. Mean, standard deviation, variance and bound values for median $\left(\widetilde{x}_{t}\right)$ for class 0 (non-spike-and-wave) and class 1 (spike-and-wave).

\begin{tabular}{lrccc}
\hline & Mean & std & Variance & Bounds \\
\hline Class 0 & $1.089 \times 10^{3}$ & $1.002 \times 10^{4}$ & $1.004 \times 10^{8}$ & {$\left[-2.769 \times 10^{4}, 2.179 \times 10^{4}\right]$} \\
Class 1 & $-6.125 \times 10^{3}$ & $2.672 \times 10^{4}$ & $7.140 \times 10^{8}$ & {$\left[-7.325 \times 10^{4}, 7.406 \times 10^{4}\right]$} \\
\hline
\end{tabular}

To illustrate the above point, Figure 4 shows a 3D scatter plot of the feature vector for spike-and-wave events (SWD, class 1, red dots) and non-spike-and-wave events (non-SWD, class 0 , blue dots). One observes that the SWD events tend to be more dispersed compared to non-SWD events. This is corroborated by Figure 5 that shows the parameters in pairs, with the following combinations

1. Scale parameter $\left(\varsigma_{t}\right)$ vs. variance $\left(\sigma_{t}^{2}\right)$ : for class 1 (SWD), one observes a direct relationship between the variance and sigma, where both parameters grow proportionally. For class 0 (non-SWD), both sigma and variance remain in a limited range of values.

2. Scale parameter $\left(\varsigma_{t}\right)$ vs. median $\left(\widetilde{x}_{t}\right)$ : as sigma grows, median increases then decreases for both SWD and non-SWD, but is larger for SWD. A cone-shaped pattern can be observed.

3. Variance $\left(\sigma_{t}^{2}\right)$ vs. median $\left(\widetilde{x}_{t}\right)$ : as the variance grows, the median increases then decreases for SWD, while it remains in a small range (cluster) for non-SWD.

The performance of our 10-nearest neighbor classifier was evaluated using a dataset consisting of 96 samples, separate from the training set. These samples were extracted from six EEG signals from subjects different from those used for training. We assessed the total accuracy of the classification. The proposed method achieved a $95 \%$ sensitivity (true positive rate), $87 \%$ specificity (true negative rate), and $92 \%$ accuracy.

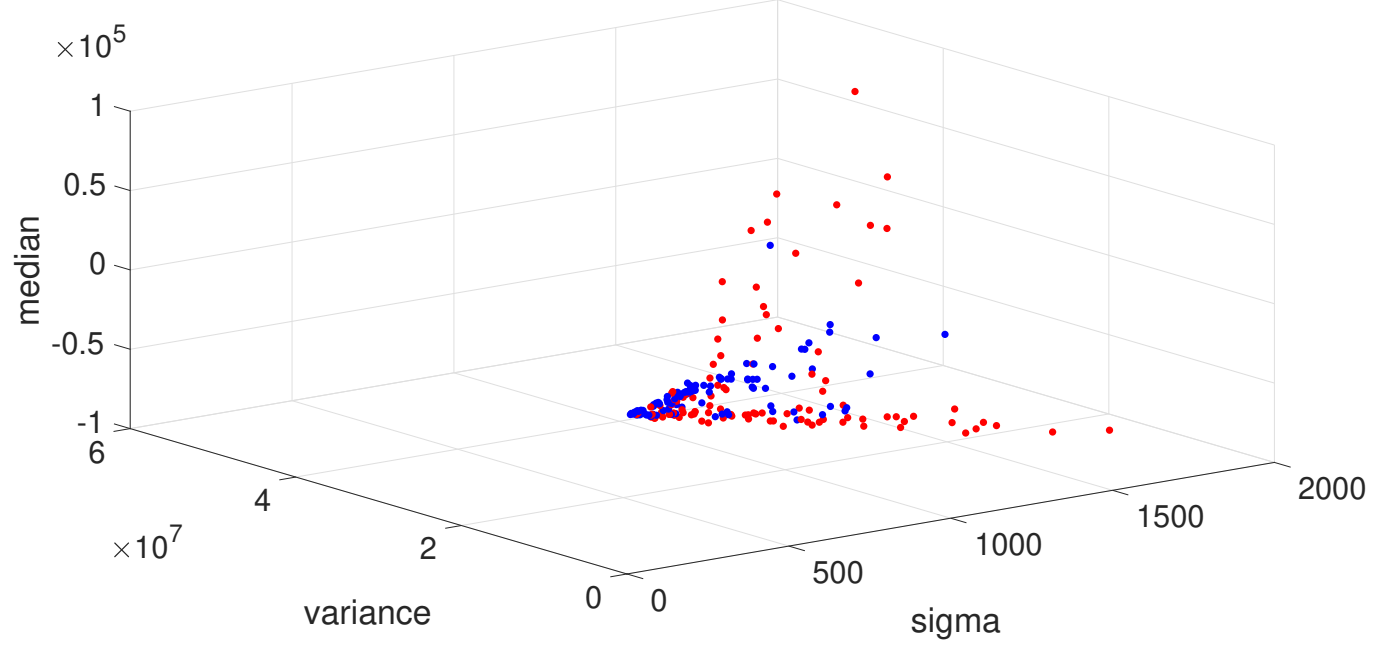

Figure 4. 3D scatter plot of the feature vector $\left[\varsigma, \sigma_{t}^{2}, \widetilde{x}_{t}\right]$ for class 0 (non-spike-and-waves events, blue dots), and class 1 (spike-and-waves events, red dots). The SWD events tend to be more dispersed than non-SWD events. 


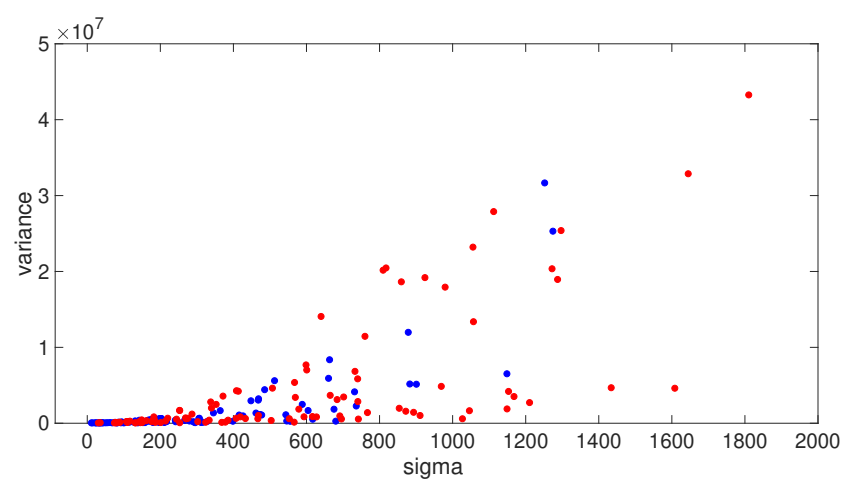

(a) sigma $\left(\varsigma_{t}\right)$ vs variance $\left(\sigma_{t}^{2}\right)$

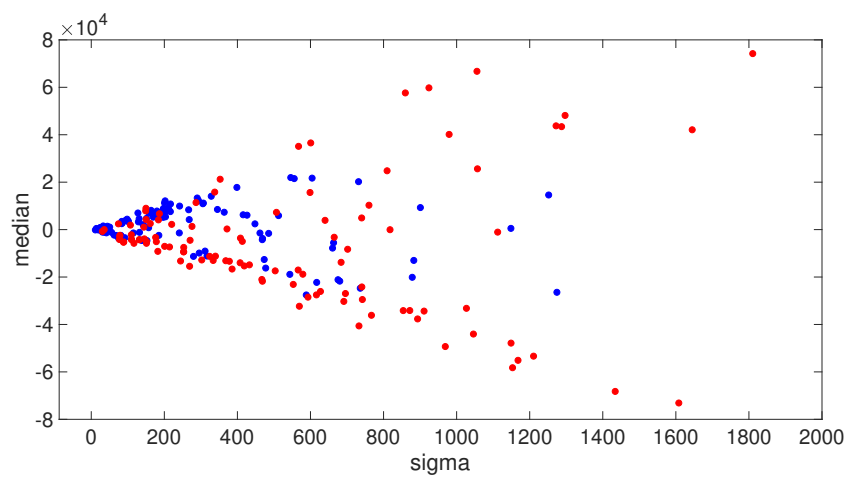

(b) sigma $\left(\varsigma_{t}\right)$ vs median $\left(\widetilde{x}_{t}\right)$

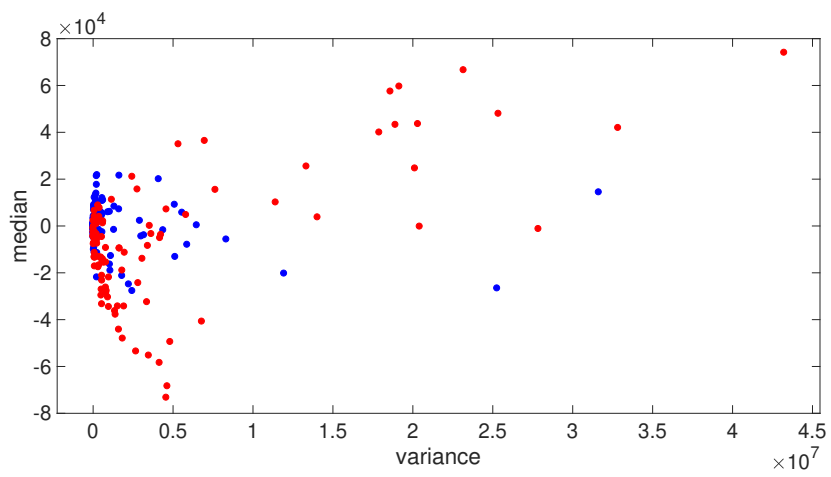

(c) variance $\left(\sigma_{t}^{2}\right)$ vs median $\left(\widetilde{x_{t}}\right)$

Figure 5. Scatter plots of the signals used for training, with $\zeta_{t}, \sigma_{t}^{2}$, and $\widetilde{x}_{t}$ parameters class 0 (non-spike-and-waves events, blue dots), and class 1 (spike-and-waves events, red dots), showing the data dispersion of the proposed approach. In (a) Scale parameter $\left(\zeta_{t}\right)$ vs. variance $\left(\sigma_{t}^{2}\right)$. For class 1 (SWD), we can see the direct relationship between the variance and sigma, both grow proportionally, while for class 0 (non-SWD) both sigma and variance remain in a range of values. (b) Scale parameter $\left(\varsigma_{t}\right)$ vs. median $\left(\widetilde{x}_{t}\right)$. As sigma grows, the median increases then decreases for both SWD and non-SWD, but is larger for SWD. (c) variance $\left(\sigma_{t}^{2}\right)$ vs. median $\left(\widetilde{x_{t}}\right)$. As variance grows, the median increases then decreases for SWD, while it remains in a small range for non-SWD.

\section{Discussion}

The proposed model-based classification method to detect patient-specific spike-and-wave events in long-term EEG signals is based on three feature parameters (or predictors). These are the scale parameter from the generalized Gaussian distribution, see Equation (6), the variance and the median, all estimated from the continuous Morlet coefficients. These features are used with a 10-nearest neighbors classifier to discriminates spike-and-wave from non-spike-and-wave events. Experimental 
results with real data from a hospital achieved $9 \%$ sensitivity (true positive rate), $87 \%$ specificity (true negative rate), and $92 \%$ accuracy. Based on our rule to choose $k$, the value was $\sqrt{212} \approx 14$, but we found a better performance by choosing empirically $k=10$. Techniques used in this study are widely known in the scientific community, but they have never before been put together to detect patient-specific epileptiform patterns in EEG. Our main contribution lies in the type of features proposed to detect spike-and-wave patterns and its application to human data. From a technical point of view, the GGD scale parameter depends on the shape parameter, see Equation (5) and Tables 2-4. They can therefore not be used together as features. Using only the scale parameter would restrict the representation space leading to pour representation of natural variability in the data. We, therefore, augmented the representation space by considering the variance and the median of wavelet coefficients. This choice has proven pertinent to discriminating SWD patterns from non-SWD.

The data collection protocol consisted of a neurologist selecting ten SWD patterns for each patient to be part of the training database. Our hypothesis was that using multiple signal patterns from individual patients improves the classification. This enhances learning patient-specific patterns, leading to precise detection of epileptiform patterns compared to previous work [39].

The collected dataset was previously used with other methods (see Table 5). We can see that the proposed method doesn't provide significantly more precise results. However, it has the advantage of analyzing the EEG signal in the time-frequency domain, where previous methods were based on temporal waveform characterization. On the other hand, the assumption that the data has a generalized Gaussian distribution allows a strong dimension reduction, leading to low computational solutions relying on rigorous statistical properties.

Table 5. Results form other methods applied to the same dataset in percent (\%), in terms of TPR = True Positives Rate or Sensitivity; TNR = True Negative Rate or Specificity; FPR = False positive Rate; ACC = Accuracy: and high Specificity, rule in (SpPIn). Training and testing have different numbers of patients due to different research settings.

\begin{tabular}{llccccccc}
\hline Method & Features & Classifier & TPR & TNR & ACC & Training & Testing & Ref. \\
\hline GGD & $\begin{array}{l}\text { GGD parameters, variance and } \\
\text { median from time-frequency } \\
\text { Morlet wavelet decomposition }\end{array}$ & $10-\mathrm{NN}$ & 95 & 87 & 92 & 212 & 96 & Actual \\
\hline $\begin{array}{l}\text { Kendall's Tau-b } \\
\text { Coefficient }\end{array}$ & $\begin{array}{l}\text { Kendall's Tau-b coefficient } \\
\text { significance in time domain }\end{array}$ & SpPIn & - & 94 & 94 & 300 & 300 & {$[39]$} \\
\hline TLS & TLS parameters in time domain & 1-NN & 100 & 100 & 100 & 192 & 46 & {$[20]$} \\
\hline Cross-correlation & $\begin{array}{l}\text { Correlation coefficient in time } \\
\text { domain }\end{array}$ & Decision trees & 86 & 98 & 97 & 96 & 46 & {$[9]$} \\
\hline
\end{tabular}

\section{Conclusions}

This paper presented a new model-based classification method to detect spike-and-wave events in long-term EEG signals in humans. The proposed method is based on the scale parameter of the generalized Gaussian distribution augmented with the variance and the median of the continuous Morlet wavelet coefficients from EEG data and a $k$-nearest neighbors classification technique.

The performance of the method was evaluated by training the model with an annotated real dataset containing 212 signal recordings consisting of spike-and-wave and non-spike-and-wave events. The classification performance was assessed by utilizing 96 segments and achieved $95 \%$ sensitivity (true positive rate), $87 \%$ specificity (true negative rate), and $92 \%$ accuracy. These results set the path to potentially new research to study the causes underlying the so-called absence epilepsy in long-term EEG recordings.

In addition to its performance, the proposed method can be implemented in online epilepsy care applications. However, due to the high dynamics of the EEG epileptic signals, some waveform might be incomplete (with part of the signal missing due to artifacts). Our method is not able to detect such situations, as confirmed by physicians using visual inspection [39]. Future work will focus on other 
epileptic waveform patterns as well as on the extensive evaluation of the proposed approach and its comparison with other methods from the literature both in humans and rodents. Other techniques, such as visual data analysis with t-distributed stochastic neighbor embedding [51] and deep learning variational autoencoders [52] will be considered. For future clinical research, an on-line user interface will be implemented with different functionalities such as automatic SWD detection and SWD pattern counts for each brain region.

Author Contributions: conceptualization, A.Q.-R., V.M. and H.B.; formal analysis, A.Q.-R. and H.B.; investigation, A.Q.-R., V.M. and H.B.; methodology, A.Q.-R. and J.P.; software, A.Q.-R.; validation, A.Q.-R., V.M., C.D. and H.B.; visualization, A.Q.-R., V.M., C.D. and H.B.; writing-original draft, A.Q.-R. and J.P.; writing-review and editing, A.Q.-R., V.M., C.D., J.P. and H.B. All authors have read and agreed to the published version of the manuscript.

Funding: This research received no external funding.

Acknowledgments: Part of the work was conducted by AQR during his Ph.D. work at Buenos Aires Institute of Technology (ITBA). We would also like to thank the Bioengineering Ivana Zorgno for her assistance in the writing.

Conflicts of Interest: The authors declare no conflict of interest.

\section{Abbreviations}

The following abbreviations are used in this manuscript:

EEG Electroencephalography

FLENI Fight against Pediatric Neurological Disease

GGD Generalized Gaussian distribution

$k$-NN $\quad k$-nearest neighbors

SWD Spike-and-wave discharge

\section{References}

1. Schomer, D.L.; da Silva, F.H.L. Niedermeyer's Electroencephalography Basic Principles, Clinical Applications, and Related Fields; LWW: Philadelphia, PA, USA, 2010.

2. Bergstrom, R.; Choi, J.; Manduca, A.; Shin, H.; Worrell, G.; Howe, C. Automated identification of multiple seizure-related and interictal epileptiform event types in the EEG of mice. Sci. Rep. 2013, 3, 1483. [CrossRef] [PubMed]

3. Bhuyan, R.; Jahan, W.; Upadhyaya, N. Interictal wave pattern study in EEG of epilepsy patients. Int. J. Res. Med. Sci. 2013, 5, 3378-3384. [CrossRef]

4. Quintero-Rincón, A.; Pereyra, M.; D'Giano, C.; Batatia, H.; Risk, M. A new algorithm for epilepsy seizure onset detection and spread estimation from EEG signals. J. Phys. Conf. Ser. 2016, 705, 12032. [CrossRef]

5. Gajic, D.; Djurovic, Z.; Gennaro, S.D.; Gustafsson, F. Classification of EEG signals for detection of epileptic seizures based on wavelets and statistical pattern recognition. Biomed. Eng. Appl. Basis Commun. 2014, 26, 1450021. [CrossRef]

6. Navakatikyan, M.; Colditz, P.; Burke, C.; Inder, T.; Richmond, J.; Williams, C. Seizure detection algorithm for neonates based on wave-sequence analysis. Clin. Neurophysiol. 2006, 117, 1190-1203. [CrossRef]

7. Siuly, S.; Kabir, E.; Wang, H.; Zhang, Y. Exploring sampling in the detection of multicategory EEG signals. Comput. Math. Methods Med. 2015, 2015, 576437.10.1155/2015/576437. [CrossRef]

8. Subasi, A.; Ercelebi, E. Classification of EEG signals using neural network and logistic regression. Comput. Methods Programs Biomed. 2005, 78, 87-99. [CrossRef]

9. Quintero-Rincón, A.; Alanis, M.; Muro, V.; D’Giano, C. Spike-and-Wave detection in epileptic signals using cross-correlation and decision trees. Rev. Argent. BioingenierÍa Bioeng. Argent. Soc. 2018, 21, 1-4.

10. Donos, C.; Dumpelmann, M.; Schulze-Bonhage, A. Early Seizure Detection Algorithm Based on Intracranial EEG and Random Forest Classification. Int. J. Neural Syst. 2015, 5, 1550023. [CrossRef]

11. Fu, R.; Tian, Y.; Shi, P.; Bao, T. Automatic Detection of Epileptic Seizures in EEG Using Sparse CSP and Fisher Linear Discrimination Analysis Algorithm. J. Med. Syst. 2020, 4, 1-13. [CrossRef]

12. Ossadtchi, A.; Greenblatt, R.E.; Towle, V.L.; Kohrman, M.H.; Kamada, K. Inferring spatiotemporal network patterns from intracranial EEG data. Clin. Neurophysiol. 2010, 121, 823-835. [CrossRef] 
13. Wilson, S.B.; Emerson, R. Spike detection: A review and comparison of algorithms. Clin. Neurophysiol. 2002, 113, 1873-1881. [CrossRef]

14. Hese, P.V.; Martens, J.; Waterschoot, L.; Boon, P.; Lemahieu, I. Automatic detection of spike and wave discharges in the EEG of genetic absence epilepsy rats from Strasbourg. IEEE Trans. Biomed. Eng. 2009, 56, 706-717. [CrossRef] [PubMed]

15. Pearce, P.; Friedman, D.; Lafrancois, J.; Iyengar, S.; Fenton, A.; Maclusky, N.; Scharfman, H. Spike wave discharges in adult Sprague Dawley rats and their implications for animal models of temporal lobe epilepsy. Epilepsy Behav. 2014, 32, 121-131. [CrossRef] [PubMed]

16. M Le Van Quyen, J.F.; Lachaux, J.; Rodriguez, E.; Lutz, A.; Martinerie, J.; Varela, F.J. Comparison of Hilbert transform and wavelet methods for the analysis of neuronal synchrony. J. Neurosci. Methods 2001, 111, 83-98. [CrossRef]

17. Puspita, J.W.; Gunadharma, A.I.J.S. Classification of epileptiform and wicket spike of EEG pattern using backpropagation neural network. AIP Conf. Proc. 2017, 1825, 020018.

18. Gupta, A.; Singh, P.; Karlekar, M. A Novel Signal Modeling Approach for Classification of Seizure and Seizure-Free EEG Signals. IEEE Trans. Neural Syst. Rehabil. Eng. 2018, 5, 925-935. [CrossRef]

19. Quintero-Rincón, A.; D'Giano, C.; Batatia, H. A quadratic linear-parabolic model-based EEG classification to detect epileptic seizures. J. Biomed. Res. 2020, 3, 203-210. [CrossRef]

20. Quintero-Rincón, A.; Prendes, J.; Muro, V.; D'Giano, C. Study on Spike-and-wave detection in epileptic signals using t-location-scale distribution and the k-nearest neighbors classifier. IEEE URUCON Congr. Electron. Electr. Eng. Comput. 2017, 2017,1-4.

21. Li, P.; Karmakar, C.; Yearwood, J.; Venkatesh, S.; Palaniswami, M.; Liu, C. Detection of epileptic seizure based on entropy analysis of short-term EEG. PLoS ONE 2018, 3, e0193691. [CrossRef]

22. Jirka, J.; Prauzek, M.; Krejcar, O.; Kuca, K. Automatic epilepsy detection using fractal dimensions segmentation and GP-SVM classification. Neuropsychiatr. Dis. Treat. 2018, 14, 2439-2449. [CrossRef]

23. Paul, Y. Various epileptic seizure detection techniques using biomedical signals: A review. Brain Inform. 2018, 5, 1-19. [CrossRef] [PubMed]

24. Subasi, A.; Alkana, A.; Koklukayab, E.; Kiymik, M.K. Analysis of Epileptic Seizure. Detection Methods Based on Parameter Estimation, Power Spectrum Density And Morlet Wavelet Transform. Neural Netw. 2005, 18, 985-997. [CrossRef]

25. Xanthopoulos, P.; Liu, C.C.; Zhang, J.; Miller, E.R.; Nair, S.P.; Uthman, B.M.; Kelly, K.; Pardalos, P.M. A robust spike and wave algorithm for detecting seizures in a genetic absence seizure model. In Proceedings of the Annual International Conference of the IEEE Engineering in Medicine and Biology Society, Minneapolis, MN, USA, 3-6 September 2009; pp. 2184-2187.

26. Sitnikova, E.; Hramov, A.E.; Koronovsky, A.A.; van Luijtelaar, G. Sleep spindles and spike-wave discharges in EEG: Their generic features, similarities and distinctions disclosed with Fourier transform and continuous wavelet analysis. J. Neurosci. Methods 2009, 180, 304-316. [CrossRef]

27. Richard, C.D.; Tanenbaum, A.; Audit, B.; Arneodo, A.; Khalil, A.; Frankel, W. SWDreader A Wavelet-Based Algorithm Using Spectral Phase to Characterize Spike-Wave Morphological Variation in Genetic Models of Absence Epilepsy. J. Neurosci. Methods 2014, 242, 127-140. [CrossRef]

28. Pollen, D.A. Intracellular studies of cortical neurons during thalamic induced wave and spike. Electroencephalogr. Clin. Neurophysiol. 1964, 17, 398-404. [CrossRef]

29. Ovchinnikov, A.; Luttjohann, A.; Hramov, A.; van Luijtelaar, G. An algorithm for real-time detection of spike-wave discharges in rodents. J. Neurosci. Methods 2010, 94, 172-178. [CrossRef] [PubMed]

30. Rodgers, K.; Dudek, F.; Barth, D. 2. Progressive, Seizure-Like, Spike-Wave Discharges Are Common in Both Injured and Uninjured Sprague-Dawley Rats: Implications for the Fluid Percussion Injury Model of Post-Traumatic Epilepsy. J. Neurosci. 2015, 35, 9194-9204. [CrossRef]

31. Blumenfeld, H. Cellular and Network Mechanisms of Spike-Wave Seizures. Epilepsia 2005, 46, 21-33. [CrossRef] [PubMed]

32. Avoli, M. A brief history on the oscillating roles of thalamus and cortex in absence seizures. Epilepsia 2012, 53, 779-789. [CrossRef]

33. Puspita, J.W.; Gunadharma, S.; Indratno, S.W.; Soewono, E. Bayesian approach to identify spike and sharp waves in EEG data of epilepsy patients. Biomed. Signal Process. Control 2017, 35, 63-69. [CrossRef] 
34. Zhu, J.D.; Lin, C.F.; Chang, S.H.; Wang, J.H.; Peng, T.I.; Chien, Y.Y. Analysis of spike waves in epilepsy using Hilbert-Huang transform. J. Med. Syst. 2014, 39, 1-13. [CrossRef] [PubMed]

35. Medvedeva, T.M.; Sysoeva, M.V.; Luijtelaa, G.; Sysoev, I.V. Modeling spike-wave discharges by a complex network of neuronal oscillators. Arch. Psychiatry Clin. Neurosci. 2018, 98, 271-282. [CrossRef]

36. Olejarczyk, E.; Rudner, R.; Marciniak, R.; Wartak, M.; Stasiowski, M.; Jalowiecki, P.; Sobieszek, A. Detection of the EEG spike-wave patterns evoked by volatile anaesthetics. IFMBE Proc. 2009, 25, 407-409.

37. Zibrandtsen, I.C.; Nielsen, J.M.; Kjaer, T.W. Quantitative characteristics of spike-wave paroxysms in genetic generalized epilepsy. Clin. Neurophysiol. 2020, 131, 1230-1240. [CrossRef]

38. Haghighi, H.S.; Markazi, A.H. Dynamic origin of spike and wave discharges in the brain. NeuroImage 2019, 197, 69-79. [CrossRef]

39. Quintero-Rincón, A.; Carenzo, C.; Ems, J.; Hirschson, L.; Muro, V.; D’Giano, C. Spike-and-wave epileptiform discharge pattern detection based on Kendall's Tau-b Coefficient. Appl. Med. Inform. 2019, 1, 1-8.

40. Tenneti, S.V.; Vaidyanathan, P.P. Absence Seizure Detection Using Ramanujan Filter Banks. In Proceedings of the 52nd Asilomar Conference on Signals, Systems, and Computers, Pacific Grove, CA, USA, 28-31 October 2018; pp. 1913-1917.

41. Polivannyi, F.; Igasaki, T.; Neshiger, N.M.R. Wavelet Transform-Based Algorithm for Single Spike-and-Wave Discharges Detection in Epileptic Patients' Electroencephalogram. In Proceedings of the 8th International Conference on BioMedical Engineering and Informatics, Shenyang, China, 14-16 October 2015; pp. 255-259.

42. Quintero-Rincón, A.; Prendes, J.; Pereyra, M.; Batatia, H.; Risk, M. Multivariate Bayesian Classification of Epilepsy EEG Signals. In Proceedings of the IEEE 12th Image, Video, and Multidimensional Signal Processing Workshop (IVMSP), Bordeaux, France, 11-12 July 2016; pp. 1-5.

43. Quintero-Rincón, A.; Pereyra, M.; D'Giano, C.; Batatia, H.; Risk, M. A visual EEG epilepsy detection method based on a wavelet statistical representation and the Kullback-Leibler divergence. In IFMBE Proceedings; Springer: Singapore, 2017; Volume 60, pp. 13-16.

44. Quintero-Rincón, A.; Pereyra, M.; D'Giano, C.; Risk, M.; Batatia, H. Fast statistical model-based classification of epileptic EEG signals. Biocybern. Biomed. Eng. 2018, 38, 877-889. [CrossRef]

45. Ahuja, N.; Lertrattanapanich, S.; Bose, N. Properties determining choice of mother wavelet. IEE Proc. Vis. Image Signal Process. 2005, 152, 659-664. [CrossRef]

46. Abry, P. Ondelettes et Turbulence. Multirésolutions, Algorithmes de Décomposition, Invariance D'échelles; Diderot Editeur: Paris, France, 1997.

47. Do, M.N.; Vetterli, M. Wavelet-Based Texture Retrieval Using Generalized Gaussian Density and Kullback-Leibler Distance. IEEE Trans. Image Process. 2002, 11, 146-158. [CrossRef]

48. Quintero-Rincón, A.; D'Giano, C.; Risk, M. Epileptic seizure prediction using Pearson's product-moment correlation coefficient of a linear classifier from generalized Gaussian modeling. Neurol. Argent. 2018, 10, 201-217.

49. Bishop, C.M. Pattern Recognition and Machine Learning (Information Science and Statistics); Springer: New York, NY, USA, 2006.

50. Barber, D. Bayesian Reasoning and Machine Learning; Cambridge University Press: Cambridge, UK, 2012.

51. van der Maaten, L.; Hinton, H.. Visualizing Data using t-SNE. J. Mach. Learn. Res. 2008, 9, 2579-2605.

52. Zemouri, R.; Lévesque, M.; Amyot, N.; Hudon, C.; Kokoko, O. Deep Variational Autoencoder: An efficient tool for PHM frameworks. In Proceedings of the Prognostics and Health Management Conference (PHM-Besancon), Besancon, France, 4-7 May 2020; pp. 235-240.

Publisher's Note: MDPI stays neutral with regard to jurisdictional claims in published maps and institutional affiliations.

(C) 2020 by the authors. Licensee MDPI, Basel, Switzerland. This article is an open access article distributed under the terms and conditions of the Creative Commons Attribution (CC BY) license (http:/ / creativecommons.org/licenses/by/4.0/). 